Revue d'histoire de l'Amérique française

REVUE D.HISTOIRE DE L'AMÉRIQUE FRANÇAISE

\title{
The Canadian Journal of Economics and Political Science, 19 (août 1953). Revue de la Canadian Political Science Association. University of Toronto Press. \$1.25.
}

\section{Michel Brunet}

Volume 7, numéro 2, septembre 1953

URI : https://id.erudit.org/iderudit/301602ar

DOI : https://doi.org/10.7202/301602ar

Aller au sommaire du numéro

Éditeur(s)

Institut d'histoire de l'Amérique française

ISSN

0035-2357 (imprimé)

1492-1383 (numérique)

Découvrir la revue

Citer ce compte rendu

Brunet, M. (1953). Compte rendu de [The Canadian Journal of Economics and Political Science, 19 (août 1953). Revue de la Canadian Political Science Association. University of Toronto Press. \$1.25.] Revue d'histoire de l'Amérique française, 7(2), 300-305. https://doi.org/10.7202/301602ar d'utilisation que vous pouvez consulter en ligne.

https://apropos.erudit.org/fr/usagers/politique-dutilisation/ 


\section{Revue des Revues}

The Canadian Journal of Economics and Political Science, 19 (août 1953). Revue de la Canadian Political Science Association. University of Toronto Press. \$1.25.

Cette revue et cette association ont exercé et continuent d'exercer une grande influence au Canada anglais. Depuis 20 ans, le Canadian Journal of Economics and Political Science a été la Bible des grands commis et des hommes d'État qui ont inspiré et dirigé la politique du gouvernement fédéral. Quiconque s'intéresse à l'histoire politique, économique, sociale et intellectuelle du Canada anglais trouvera dans les volumes de cette revue une documentation d'une importance capitale. Lire le Canadian Journal of Economics and Political Science est plus révélateur que de dépouiller les Débats de la Chambre des Communes. Les idées et les programmes des chefs politiques ont d'abord été lancés et exposés par les membres de la Canadian Political Science Association et par les collaborateurs du Canadian Journal of Economics and Political Science. Si les historiens, les sociologues, les économistes, les journalistes et les hommes politiques du Canada français s'étaient imposé la lecture régulière et attentive de cette revue, ils auraient peut-être mieux compris l'évolution contemporaine du Canada anglais. Ils préféraient s'abonner à la Revue des Deux Mondes et à d'autres publica- 
tions outre-atlantiques. Celles-ci ont leur mérite, mais elles n'apprendront jamais à un Canadien français ce qu'il doit savoir sur son pays. C'est pourquoi nous rencontrons tant de Canadiens français capables de disserter pertinemment sur la politique intérieure de la France mais qui ignorent le rapport MacMillan, l'enquête sur les relations fédérales-provinciales et le rapport Marsh. Ils prennent part à tous les débats entre partisans du maréchal Pétain et défenseurs de la Troisième République. Ne leur demandez pas, cependant, s'ils ont lu le rapport Massey. Cette situation plutôt ridicule a suffisamment duré. Il n'est nullement question de nier ou de rejeter le magistère intellectuel de la France. Il s'agit tout simplement de devenir adultes et de reconnaître qu'il existe des domaines où la France n'a rien à nous apprendre. Il est urgent de nous en rendre compte, particulièrement dans le domaine des sciences politiques et économiques. Un compte rendu critique du dernier numéro donnera une idée de la riche matière offerte habituellement aux lecteurs du Canadian Journal of Economics and Political Science.

La conference de M. Herbert Marshall, "The Role of the Dominion Bureau of Statisties in the Post-War World" (281-290), démontre l'importance du service de la statistique dans l'élaboration de la politique économique et sociale du gouvernement central. En plusieurs milieux, on persiste à ridiculiser les travaux et les recherches des statisticiens. Cette attitude dénote chez ceux qui l'adoptent une ignorance impardonnable des problèmes contemporains. Ceux qui riront les derniers ne seront pas les adversaires de la statistique. Les statisticiens fournissent aux gouvernements, aux financiers, aux directeurs de la grande industrie et du grand commerce, des renseignements indispensables. Sans la statistique, notre civilisation industrielle avancerait à l'aveuglette. Le gouvernement d'Ottawa l'a depuis longtemps compris et il s'est donné un service de statistique dont la compétence est reconnue universellement.

Le professeur W.T. Easterbrook de l'Université de Toronto étudie la pensée d'un historien et d'un économiste qui a tenu une place de premier plan dans le monde universitaire anglo-canadien, "Harold Innis and Economics" (291-303). Il est intéressant de suivre pas à pas l'itinéraire intellectuel d'un chercheur consciencieux et original qui a tenté de s'expliquer le monde dans lequel il vivait. Son explication ne s'inspirait pas de préconceptions arbitraires mais représentait les conclusions d'une recherche patiente et désintéressée. C'est la seule fạ̧on de procéder dans le domaine de la science empirique.

Deux articles résument très bien le problème des relations fédéralesprovinciales et les objectifs de la politique économico-sociale du gouvernement central: W.J. Waines, "Dominion-Provincial Financial Arrangements: An Examination of Objectives" (304-315); Monteath Douglas, "Welfare and Distribution: Economic Implications of the Redistributive Processes Involved in Canadian Social Welfare Policies" (316-325). Il est impossible d'aborder l'étude des relations fédérales-provinciales sans tenir comptè du 
rôle de plus en plus important assumé par l'État fédéral dans la mise en vigueur d'une politique à l'échelle nationale. De 1918 à 1930, le gouvernement d'Ottawa a connu une période de stagnation: l'ère de la construction des chemins de fer transcontinentaux était terminée et la fin de la guerre avait enlevé l'initiative au gouvernement central. Celui-ci se cherchait de nouvelles sphères d'action afin de redevenir le "grand gouvernement" qu'il avait été au début de la Confédération. Les provinces profitèrent de cette éclipse temporaire du gouvernement fédéral. Pendant quelques années, elles se donnèrent l'illusion d'être les plus fortes. Elles en vinrent à croire qu'elles avaient créé le gouvernement d'Ottawa et que celui-ci était leur serviteur. Les décisions du Conseil privé en faveur de l'autonomie provinciale avaient encouragé cet excès de confiance. La prospérité apportée par l'exploitation des ressources naturelles - forêts, pouvoirs d'eau et mines et l'expansion industrielle maintinrent cette "grande illusion" de l'histoire constitutionnelle canadienne. La crise économique de 1929 y mit brutalement fin. Depuis, le gouvernement fédéral a pris l'initiative dans tous les domaines. Rien n'indique son intention de se retirer pour laisser la place aux gouvernements provinciaux. Les deux auteurs, adoptant l'attitude de l'immense majorité au Canada anglais - on pourrait même dire que l'unanimité est faite à ce sujet, approuvent cette évolution vers une politique de centralisation intégrale destinée à réduire les provinces à de simples rouages administratifs. M. Douglas ne peut s'empêcher, cependant, de souligner les faiblesses de la loi nationale des pensions de vieillesse.

L'article de Norman Ward, "The Redistribution of 1952" (341-360), raconte la grande inquiétude qui s'empare des députés lorsque vient l'heure de redistribuer les circonscriptions électorales. L'auteur croit que le Parlement finira par confier cette tâche à une commission indépendante. Cette réforme marquerait une autre étape vers la maturité politique. $M$. Ward rappelle que dans la plupart des provinces la population urbaine n'obtient pas la représentation à laquelle elle a droit. Cette situation fausse le mécanisme de nos institutions démocratiques.

L'étude de M.P. O'Connell, "The Ideas of Henri Bourassa" (361-376) intéressera tous ceux qui cherchent à comprendre la carrière publique d'un homme qui a épousé plusieurs causes. M. O'Connell avoue qu'il n'est pas facile d'expliquer la personnalité complexe de Bourassa. Celui-ci avait un tempérament très violent. Son éloquence lui inspira fréquemment des déclarations à l'emporte-pièce qui outrepassaient sa pensée. Il résistait difficilement à la tentation de scandaliser les faibles. Avouons que ses compatriotes lui en fournirent souvent l'occasion. L'auteur n'ignore pas ces faits et cherche à dégager les notes dominantes et les lignes de force de la pensée bourassiste en tenant compte de l'homme et du milieu dans lequel celui-ci a évolué.

Trois problèmes vitaux sollicitaient l'attention inquiète de l'élite canadienne-française au début du XXe siècle: la participation plus directe du Canada à la vie impériale et internationale, l'immigration et le développoment de l'Ouest, le bouleversement économico-social apporté au Québec 
traditionnel par l'industrialisation rapide de la province. Il ne faudrait pas croire que ce dernier phénomène est tout récent. Trop de personnes s'imaginent que la province de Québec a commencé à s'industrialiser en 1943. Comment Bourassa réagit-il devant ces problèmes?

En politique extérieure, même s'il s'intéressa toujours de très près aux relations internationales, il demeura toute sa vie un neutraliste et un pacifiste. Il appartenait à la tradition isolationiste nord-américaine édifiée à l'abri de la doctrine Monroe et de la flotte britannique au cours du XIXe siècle. La situation avait pourtant bien changé depuis l'avènement de l'Allemagne impériale et impérialiste! L'immense majorité des Canadiens anglais s'en était rendu compte et était prête à faire sa part pour la défense de l'Empire. Deux guerres mondiales l'ont prouvé.

En politique intérieure, Bourassa prêcha un large canadianisme dans lequel se fondraient les immigrants et les Canadiens anglophones et francophones. Les bases de ce canadianisme seraient l'autonomie des provinces et l'établissement d'un État national biculturel. L'émancipation politique graduelle du Canada à l'intérieur des cadres de l'Empire britannique favoriserait l'éclosion de ce canadianisme at large. L'auteur soutient que Bourassa ne se déclara jamais en faveur de la rupture du lien impérial et de la fondation, d'une république canadienne. Ce canadianisme dont rêvait Bourassa semble en voie de formation. Seul l'avenir dira si le Canada peut être autre chose qu'un royaume anglais ou un État annexé aux États-Unis.

Alarmé devant les conséquences économico-sociales de l'industrialisation du Québec par le capital étranger, il préconisa, en unisson avec tous les chefs religieux et laïques de la minorité canadienne-française, une politique intensive de colonisation agricole, une utilisation plus rationnelle et plus lucrative des ressources naturelles de la province et une législation visant à protéger la petite entreprise et la petite propriété. Il partageait tous les préjugés de son milieu contre les grandes affaires et voyait le salut de ses compatriotes dans le maintien d'une société de petits propriétaires terriens, de petits industriels et de petits marchands. Il était le porte-parole des classes moyennes du Canada français. Celles-ci se sentaient livrées sans défense à l'invasion triomphante de la haute finance et de la grande industrie anglo-saxonnes. Bourassa fut l'un des premiers à deviner, jusqu'à un certain point, les services que pourraient rendre aux siens le syndicalisme catholique et le mouvement coopératif. Même s'il avait été intégralement appliqué, le programme de Bourassa et de ses disciples n'aurait pas prévenu la prolétarisation massive des Canadiens français au service du capitalisme étranger anglais, américain et anglo-canadien. Le fondateur du Devoir a eu, cependant, le mérite de prévoir que les luttes de ses compatriotes, au XXe siècle, se livreraient sur le terrain économique. L'histoire récente laisse soupçonner que la deuxième partie du siècle sera témoin de conflits économico-sociaux auprès desquels les luttes politico-oratoires du siècle dernier prendront figure de débats académiques. Il serait puéril de croire que la province de Québec ne connaîtra pas les luttes de classes que la révolution industrielle 
capitaliste a suscité dans tous les pays où elle a fait brutalement sauter, sans transition, l'ancien ordre social. Le capitalisme étranger a démoli la société rurale canadienne-française du XIXe siècle. Il a imposé un ordre économicosocial que la masse canadienne-française subit mais n'accepte pas. Peu à peu, le prolétariat québécois prend conscience de sa situation. C'est la période que nous traversons actuellement. Que réserve l'avenir? Seuls les aveugles volontaires ou involontaires diront qu'il s'annonce calme et serein.

L'auteur souligne plusieurs autres aspects de la pensée de Bourassa: son catholicisme militant, son admiration pour l'école des Little Englanders dont les enseignements ont faussé sa conception de l'Empire britannique, sa méfiance à l'égard des partis politiques, son mépris aristocratique pour la démocratie, son antiétatisme, son enthousiasme quelque peu naï et même colonialiste, particulièrement au début de sa carrière, pour les institutions britanniques, son anglophobie latente qui, à certains moments, éclata au grand jour. Ce dernier trait ne doit pas surprendre. L'anglophobie est un sentiment inné chez le Canadien français. Tout comme la francophobie chez le Canadian anglais. André Siegfried avait remarqué ce fait lors de son premier voyage au Canada. Deux cents ans d'habitat commun ont simplement enseigné aux Canadiens des deux langues à taire leurs sentiments intimes d'inimitié. En public du moins. Et pas toujours, malheureusement.

L'article de M. O'Connell mérite d'être lu et relu. Il est évident, toutefois, que l'auteur n'a pas encore terminé ses recherches. Son étude donne l'impression d'une ébauche. Il avance sur un terrain qu'il ne connaît pas encore suffisamment. La complexité du personnage dépend directement $d u$ milieu canadien-français auquel Bourassa, le petit-fils de Papineau, appartenait de toutes les fibres de son être - même quand il essayait de s'élever à un canadianisme global. Impossible de comprendre Bourassa sans connaître à fond l'histoire de la minorité canadienne-française depuis la Conquête anglaise. Si M. O'Connell se propose d'écrire une biographie de Bourassa, il devra réétudier l'histoire du Canada français dans une perspective différente de celle que l'histoire traditionnelle nous présente.

H.C. Pentland, répondant aux critiques de Hugh C.J. Aitken, complète son étude d'histoire économique publiée dans le numéro de novembre 1950. Ce premier article s'intitulait "The Role of Capital in Canadian Economic Development before 1875" (Canadian Journal of Economics and Political Science, 16 (novembre 1950): 457-474). M. Aitken avait mis en doute certaines affirmations de M. Pentland, "A Note on the Capital Resources of Upper Canada" (ibid., 18 (novembre 1952): 525-533). Le professeur Pentland, après avoir poursuivi ses recherches, donne partiellement raison à son contradicteur et apporte quelques précisions supplémentaires, "Further Observations on Canadian Development" (403-410). Ces trois articles d'histoire économique sont à lire et à relire. Ils montrent comment le Canada anglais a réussi à bâtir son économie avec l'aide du capital britannique. M. Pentland calcule que, de 1827 à 1875, la Grande-Bretagne plaça ou dépensa au Canada quelque 400 millions de dollars. Ces chiffres, beaucoup mieux que 
les hypothèses psychologiques ou autres, expliquent à quoi tient la supériorité économique des Anglo-Canadiens. Les Canadiens français, comme l'écrivait $L a$ Minerve, le 5 mars 1832, n'ont jamais eu les mêmes avantages: "Nous ne puisons pas à la même source; nous n'avons aucune protection hors du pays; nous ne nous servons pas des capitaux étrangers pour nous en créer à nous-mêmes". (Voir cet article dans Histoire du Canada par les textes, 147-148). Les Canadiens français de la première moitié du XIXe siècle n'entretenaient pas d'illusions sur leur force économique réelle. Ils savaient, de plus, comment s'édifie l'économie d'un pays d'Amérique. Leurs descendants sont moins modestes et plus ignorants.

Deux articles nous renseignent sur la carrière féconde de celui qui fut sous-ministre des Finances du Canada pendant vingt ans: W.A. Mackintosh, "William Clifford Clark and Canadian Economic Policy" (411-413), et R.B. Bryce, "William Clifford Clark, 1889-1952" (413-423). Clark occupait l'une des premières places dans l'équipe des grands commis qui ont inspiré et conseillé les chefs politiques du Canada anglais durant les vingt dernières années. L'histoire récente de notre pays, depuis la création de la Banque du Canada jusqu'au rapport Massey, ne peut pas s'écrire sans chercher à connaître le rôle exact joué par les universitaires - particulièrement les économistes, les historiens et les political scientists - et les hauts fonctionnaires anglo-canadiens. Leur influence dans la politique canadienne contemporaine se compare à celle des grands capitalistes au XIXe siècle. Cette évolution se remarque dans tous les pays du monde atlantique. Les dénonciations lancées périodiquement contre ceux que plusieurs appellent avec mépris les "bureaucrates" n'ont rien changé et ne changeront rien à cette évolution. Un État moderne ne se conçoit pas sans une bureaucratie formée de grands commis dont la compétence et la vision servent les intérêts du gouvernement qui se les attache. Au Canada, c'est le gouvernement central qui l'a compris le premier. Il ne doit pas le regretter.

D'autres articles plus spécialisés et une revue des livres complètent ce numéro du Canadian Journal of Economics and Political Science. Il n'est pas nécessaire d'insister davantage pour convaincre nos lecteurs qu'ils ont intérêt à lire cette revue importante s'ils veulent se tenir au courant de la pensée politique, économique et sociale du Canada anglais. Plus les Canadiens français se familiariseront avec la pensée anglo-canadienne, mieux ils comprendront l'histoire passée et contemporaine du Canada. Si nous négligeons de le faire, nous risquons de continuer à entretenir de grandes et stériles illusions sur l'évolution historique d'un pays où nous sommes une minorité soumise, bon gré mal gré, aux décisions de la majorité. Payonsnous au moins le luxe de savoir quel est le programme de ceux qui dirigent la politique canadienne.

Professeur agrégé,

Michel Brune T

Institut d'histoire,

Faculté des Lettres,

Université de Montréal. 